\title{
Effect of total and pair configurational entropy in determining dynamics of supercooled liquids over a range of densities
}

\author{
Atreyee Banerjee, ${ }^{1}$ Manoj Kumar Nandi, ${ }^{1}$ Srikanth Sastry, ${ }^{2}$ and Sarika Maitra Bhattacharyya ${ }^{1, a)}$ \\ ${ }^{1)}$ Polymer Science and Engineering Division, CSIR-National Chemical Laboratory, Pune-411008, \\ India \\ 2) Theoretical Sciences Unit, Jawaharlal Nehru Centre for Advanced Scientific Research, Jakkur Campus, \\ Bengaluru 560 064, India
}

(Dated: 11 October 2018)

In this paper, we present a study of supercooled liquids interacting with the Lennard Jones (LJ) potential and the corresponding purely repulsive (Weeks-Chandler-Andersen or WCA) potential, over a range of densities and temperatures, in order to understand the origin of their different dynamics in spite of their structures being similar. Using the configurational entropy as the thermodynamic marker via the Adam Gibbs (AG) relation, we show that the difference in the dynamics of these two systems at low temperatures can be explained from thermodynamics. At higher densities both the thermodynamical and dynamical difference between these model systems decrease, which is quantitatively demonstrated in this paper by calculating different parameters. The study also reveals the origin of the difference in pair entropy despite the similarity in the structure. Although the maximum difference in structure is obtained in the partial radial distribution function (rdf) of the $\mathrm{B}$ type of particles, the rdf of $\mathrm{AA}$ pairs and $\mathrm{AB}$ pairs give rise to the differences in the entropy and dynamics. This work supports the observation made in an earlier study (Phys. Rev. Lett.,113, $225701,2014)$ and shows that they are generic in nature, independent of density.

\section{INTRODUCTION}

Historically, the van der Waals picture assumes that the short range, harsh repulsive part of the potential determines the structure of the fluid and the attractive part of the potential provides a homogeneous background ${ }^{1}$. In this spirit, Weeks, Chandler and Andersen proposed that for liquids at high density, the Lennard Jones (LJ) potential can be treated as a sum of repulsive and attractive parts, and the repulsive part of the potential, commonly known as the WCA potential, is sufficient to describe the structure and the dynamics of liquids ${ }^{2}$. For a supercooled liquid with closely packed particles this picture is expected to provide a good description. However, in a series of papers Bertheir and Tarjus have shown that although the structures of the WCA and LJ systems are very close at the same temperature and density, their dynamics at low temperatures are very different ${ }^{3-6}$. They have questioned the role of thermodynamics in determining the dynamics. They have also used various theories which depend on the pair correlation function and have shown that none of the theories can differentiate the two systems $s^{4,5,7,8}$. Thus they have proposed that higher-order correlation functions or topological measure are more sensitive to capture the difference between the two systems ${ }^{6}$. Coslovich has indeed shown that triplet correlations are much more sensitive and can disentangle the two systems ${ }^{9,10}$. He has shown that the LJ system has more pronounced local ordering ${ }^{9}$. The sluggish dynamics in supercooled liquids comes from these locally preferred structures which are known to form correlated

a) Electronic mail: mb.sarika@ncl.res.in domains ${ }^{11}$. The estimation of the lengthscale of the domains is done in different ways ${ }^{12-17}$. One of them is the measurement of the point-to-set (PTS) correlation length ${ }^{16,18-20}$. Hockey et. al have shown that the difference in the static length scale (PTS) can account for the difference in the dynamics of the LJ and WCA systems. Although the connection between the PTS and dynamics does point to the role of thermodynamics, it cannot completely rule out the role of kinetic constraints ${ }^{21}$. Wang et al. have connected the glass transition temperature to the vibrational density of states and they found that above a critical density, the LJ and WCA systems behave in a similar way ${ }^{22}$. A similar connection between the two systems have also been made by looking at fragility ${ }^{23}$. Dyre and co-workers ${ }^{24-26}$ have argued that the origin of dynamical differences between LJ and WCA systems is not the neglect of the attractive part ${ }^{26}$ but rather the truncation of the WCA potential ${ }^{24}$. They have also predicted that at high densities when there is inclusion of interactions from all first neighbours, the WCA system can mimic the LJ system. Bohling et al. have also shown that the dynamics of the attractive LJ system can be mapped to a repulsive inverse power law potential proving their point that the difference in the dynamics is not due to the nature of the potential ${ }^{26}$.

In a recent work by some of us, it has been shown that the dynamics of the LJ and WCA systems follow the thermodynamics. It has been conclusively proven that the difference in the dynamics is a reflection of the difference in their thermodynamics ${ }^{27}$. The well known Adam-Gibbs relation ${ }^{28}$, which connects thermodynamics to kinetics ${ }^{29-38}$, is found to be valid for LJ and WCA systems. It has also been found that although their pair correlation functions look very similar ${ }^{3}$, the pair entropy and pair relaxation time predicted by Adam-Gibbs relax- 
ation are capable of showing the difference between the LJ and WCA systems ${ }^{27}$. However our previous study was performed at a specific density. In the present paper we have studied a wider range of densities, and show that the observations made at $\rho=1.2$ are generic. We study the density dependence of different thermodynamic and kinetic parameters such as the fragility ${ }^{39,40}$ and various transition temperatures. Here we analyse the origin of the difference in the pair entropy between the two models. We also show that although the AG relation at the two body level shows reminiscence of mode coupling theory $(\mathrm{MCT})$, it fares better in predicting the difference between the dynamics of the two systems.

The paper is organized as follows: The simulation details are given in Sec. II. In Sec. III we describe the methods used for evaluating the various quantities of in- terest and provide other necessary background. In Sec. IV we report the simulation results and their analysis. Sec. V contains a discussion of presented results and conclusions.

\section{SIMULATION DETAILS}

We have performed molecular dynamics simulations of the Kob-Andersen model which is a binary mixture (80:20) of Lennard-Jones (LJ) particles and the corresponding WCA version ${ }^{2,41}$. The interatomic pair potential between species $\alpha$ and $\beta$, with $\alpha, \beta=A, B$,

$U_{\alpha \beta}(r)$ is described by a truncated and shifted Lennard-Jones (LJ) potential, as given by:

$$
U_{\alpha \beta}(r)= \begin{cases}U_{\alpha \beta}^{(L J)}\left(r ; \sigma_{\alpha \beta}, \epsilon_{\alpha \beta}\right)-U_{\alpha \beta}^{(L J)}\left(r_{\alpha \beta}^{(c)} ; \sigma_{\alpha \beta}, \epsilon_{\alpha \beta}\right), & r \leq r_{\alpha \beta}^{(c)} \\ 0, & r>r_{\alpha \beta}^{(c)}\end{cases}
$$

where $U_{\alpha \beta}^{(L J)}\left(r ; \sigma_{\alpha \beta}, \epsilon_{\alpha \beta}\right)=4 \epsilon_{\alpha \beta}\left[\left(\sigma_{\alpha \beta} / r\right)^{12}-\left(\sigma_{\alpha \beta} / r\right)^{6}\right]$ and $r_{\alpha \beta}^{(c)}=2.5 \sigma_{\alpha \beta}$ for the LJ systems and $r_{\alpha \beta}^{(c)}$ is equal to the position of the minimum of $U_{\alpha \beta}^{(L J)}$ for the WCA systems. Length, temperature and time are given in units of $\sigma_{A A}, k_{B} T / \epsilon_{A A}$ and $\tau=\sqrt{ }\left(m_{A} \sigma_{A A}^{2} / \epsilon_{A A}\right)$, respectively. Here we have simulated the Kob-Andersen Model with the interaction parameters $\sigma_{A A}=1.0, \sigma_{A B}=0.8, \sigma_{B B}$ $=0.88, \epsilon_{A A}=1, \epsilon_{A B}=1.5, \epsilon_{B B}=0.5, m_{A}=m_{B}=1.0$.

The molecular dynamics (MD) simulations have been carried out using the LAMMPS package ${ }^{42}$. We have performed MD simulations in the canonical ensemble (NVT) using Nosé-Hoover thermostat with integration timestep $0.005 \tau$. The time constant for the Nosé-Hoover thermostat are taken to be 100 timesteps. The sample is kept in a cubic box with periodic boundary conditions. The system size is $N=500, N_{A}=400$ ( $\mathrm{N}=$ total number of particles, $N_{A}=$ number of particles of type A) and we have studied a broad range of density $\rho$ from 1.2 to 1.6. For all state points, three to five independent samples with run lengths $>100 \tau$ ( $\tau$ is the $\alpha$-relaxation time) are analyzed.

\section{DEFINITION}

\section{A. Relaxation time}

We have calculated the relaxation times from the decay of the overlap function $\mathrm{q}(\mathrm{t})$, from the condition $q(t=$ $\left.\tau_{\alpha}, T\right) / N=1 / e \cdot q(t)$ is defined as

$$
\begin{array}{r}
\langle q(t)\rangle \equiv\left\langle\int d r \rho\left(r, t_{0}\right) \rho\left(r, t+t_{0}\right)\right\rangle \\
=\left\langle\sum_{i=1}^{N} \sum_{j=1}^{N} \delta\left(\mathbf{r}_{j}\left(t_{0}\right)-\mathbf{r}_{i}\left(t+t_{0}\right)\right)\right\rangle \\
=\left\langle\sum_{i=1}^{N} \delta\left(\mathbf{r}_{i}\left(t_{0}\right)-\mathbf{r}_{i}\left(t+t_{0}\right)\right)\right\rangle \\
+\left\langle\sum_{i} \sum_{j \neq i} \delta\left(\mathbf{r}_{i}\left(t_{0}\right)-\mathbf{r}_{j}\left(t+t_{0}\right)\right)\right\rangle .
\end{array}
$$

The overlap function is a two-point time correlation function of local density $\rho(r, t)$. It has been used in many recent studies of slow relaxation ${ }^{43}$. In this work, we consider only the self-part of the total overlap function (i.e. neglecting the $i \neq j$ terms in the double summation). Earlier it has been shown to be a good approximation to the full overlap function. So, the overlap function can be well approximated by its self part, and written as,

$$
\langle q(t)\rangle \approx\left\langle\sum_{i=1}^{N} \delta\left(\mathbf{r}_{i}\left(t_{0}\right)-\mathbf{r}_{i}\left(t+t_{0}\right)\right)\right\rangle .
$$

Again, the $\delta$ function is approximated by a window function $\omega(x)$ which defines the condition of overlap between two particle positions separated by a time interval t:

$$
\begin{array}{r}
\langle q(t)\rangle \approx\left\langle\sum_{i=1}^{N} \omega\left(\left|\mathbf{r}_{i}\left(t_{0}\right)-\mathbf{r}_{i}\left(t+t_{0}\right)\right|\right)\right\rangle \\
\omega(x)=1, x \leq \text { a implying overlap } \\
=0, \text { otherwise. }
\end{array}
$$


The time dependent overlap function thus depends on the choice of the cut-off parameter a, which we choose to be 0.3 . This parameter is chosen such that particle positions separated due to small amplitude vibrational motion are treated as the same, or that $a^{2}$ is comparable to the value of the MSD in the plateau between the ballistic and diffusive regimes.

Relaxation times obtained from the decay of the self intermediate scattering function $F_{s}(k, t)$ using the definition $F_{s}\left(k, t=\tau_{\alpha}, T\right)=1 / e$ at $k \simeq 2 \pi / r_{\min }$. The self intermediate scattering function is calculated from the simulated trajectory as

$$
F_{s}(k, t)=\frac{1}{N}\left\langle\sum_{i=1}^{N} \exp \left(-i \mathbf{k} .\left(\mathbf{r}_{i}(t)-\mathbf{r}_{i}(0)\right)\right)\right\rangle .
$$

Since relaxation times from $q(t)$ and $F_{s}(k, t)$ behave very similarly at low temperature, we have used the time scale obtained from $q(t)$.

\section{B. Diffusivity}

Diffusivities (D ) are obtained from the mean squared displacement (MSD) of the particles. We have calculated MSD as follows,

$$
R^{2}(t)=\frac{1}{N} \sum_{i}\left\langle\left(\mathbf{r}_{i}(t)-\mathbf{r}_{i}(0)\right)^{2}\right\rangle
$$

and from the long time behavior of MSD, the diffusion coefficient D obtained as,

$$
D=\lim _{t \rightarrow \infty} \frac{R^{2}(t)}{6 t} .
$$

\section{Fragility}

Fragility 39,40 is a parameter which quantifies the rate at which any dynamical quantity (viscosity, relaxation times and inverse diffusivities) grows with temperature. There are many ways to quantify the fragility among which two are more popular. One is from the "steepness index" m, which is defined from the so-called Angell plot. The slope $(\mathrm{m})$, of logarithm of the viscosity $(\eta)$ or relaxation time $(\tau)$ at the laboratory glass transition temperature $T=T_{g}$, with respect to the scaled inverse temperature $\frac{T_{g}}{T}$, is written as, $m=\left(\frac{d \log \tau}{d\left(T_{g} / T\right)}\right)_{T=T_{g}}$

Another way of defining fragility is by plotting the temperature dependence of the viscosity or the relaxation time in terms of modified Vogel-Fulcher-Tammann (VFT) equation,

$$
\tau(T)=\tau_{o} \exp \left[\frac{1}{K_{V F T}\left(\frac{T}{T_{V F T}}-1\right)}\right],
$$

where $K_{V F T}$ is the kinetic marker for fragility, $T_{V F T}$ is the temperature where the relaxation time diverges and $\tau_{o}$ is the high temperature relaxation time. In this paper we have used the Vogel-Fulcher-Tammann (VFT) fits to the temperature dependence of dynamical quantities for defining kinetic fragility.

The thermodynamic fragility, on the other hand, is obtained from the temperature dependence of the configurational entropy $S_{c}$. The method of $S_{c}$ calculation will be discussed in the next subsection. The temperature dependence of $S_{c}$ is given by

$$
T S_{c}=K_{T}\left(\frac{T}{T_{K}}-1\right),
$$

where $K_{T}$ is termed as the thermodynamic marker for fragility and $T_{K}$ is the Kauzmann temperature. The correlation between the kinetic fragility and the thermodynamic fragility and thus the potential energy landscape can be drawn from the well known Adam-Gibbs (AG) relation $^{28}$,

$$
\tau(T)=\tau_{o} \exp \left(\frac{A}{T S_{c}}\right) .
$$

Here $A$ is the Adam-Gibbs parameter. The above equation predicts Arrhenius behaviour for constant $S_{c}$ and thus it relates $A$ to the high temperature activation energy $E_{\infty}{ }^{43}$. By using Eq.9 in Eq.10 the AG relation yields the VFT (Eq.8) relation with the identification of $K_{V F T} \simeq K_{A G}=K_{T} / A$ and $T_{V F T} \simeq T_{K}$.

\section{Configurational entropy}

Configurational entropy, $S_{c}$ per particle, the measure of the number of distinct local energy minima, is calculated $^{44}$ by subtracting from the total entropy of the system the vibrational component: $S_{c}(T)=S_{\text {total }}(T)-$ $S_{v i b}(T)^{30,43}$. Here the total entropy, $S_{\text {total }}=S_{i d}+S_{e x}$, is obtained by summing the ideal gas entropy $S_{i d}$ with the excess entropy $S_{e x}$ where the latter is obtained via thermodynamic integration from the ideal gas limit. Vibrational entropy for the binary mixture is calculated by making a harmonic approximation to the potential energy about a given local minimum.

\section{E. Pair configurational entropy}

As discussed earlier the excess entropy $S_{e x}$, defined as the difference between the total entropy $\left(S_{\text {total }}\right)$ and the ideal gas entropy $\left(S_{i d}\right)$ at the same temperature $(T)$ and density $(\rho)$, can also be expanded in an infinite series, $S_{e x}=S_{2}+S_{3}+\ldots . .=S_{2}+\Delta S$ using Kirkwood's factorization ${ }^{45}$ of the N-particle distribution function ${ }^{46-48}$. $S_{n}$ is the " $n$ " body contribution to the entropy. Thus the pair excess entropy is $S_{2}$ and the higher order contributions to excess entropy is given by the residual multiparticle entropy (RMPE), $\Delta S=S_{e x}-S_{2}{ }^{49-52}$. The pair entropy $S_{2}$ for a binary 
system can be written in terms of the partial radial dis- tribution functions,

$$
\frac{S_{2}}{k_{B}}=-2 \pi \rho \sum_{\alpha, \beta} x_{\alpha} x_{\beta} \int_{0}^{\infty}\left\{g_{\alpha \beta}(r) \ln g_{\alpha \beta}(r)-\left[g_{\alpha \beta}(r)-1\right]\right\} r^{2} d r
$$

where $g_{\alpha \beta}(r)$ is the atom-atom pair correlation between atoms of type $\alpha$ and $\beta, \rho$ is the density of the system, $x_{\alpha}$ is the mole fraction of component $\alpha$ in the mixture, and $k_{B}$ is the Boltzmann constant.

To get an estimate of the configurational entropy as predicted by the pair correlation we rewrite $S_{c}$ in terms of the pair contribution to configurational entropy $S_{c 2}{ }^{27}$,

$S_{c}=S_{i d}+S_{e x}-S_{v i b}=S_{i d}+S_{2}+\Delta S-S_{v i b}=S_{c 2}+\Delta S$.

Thus, the pair configurational entropy is defined as, $S_{c 2}=S_{i d}+S_{2}-S_{v i b}$. Note that an expansion of $S_{v i b}$ in terms of two and many body would be interesting but to the best of our knowledge this has not been attempted before. Thus we have used total $S_{v i b}$.

\section{F. Mode coupling theory}

Many properties of glass forming liquids can be explained by the well known mode coupling theory (MCT). This microscopic theory can give a qualitative description of dynamical properties (such as temperature dependence of relaxation time) if the static structure of the liquid is known and many experiments and simulation results have shown that MCT predictions hold good in the temperature regime of initial slow down of dynamics ${ }^{53,54}$. The equation for the intermediate scattering function for the binary mixture is given by,

$$
\ddot{\boldsymbol{S}}(k, t)+\boldsymbol{\Gamma} \dot{\boldsymbol{S}}(k, t)+\boldsymbol{\Omega}^{2}(k) \boldsymbol{S}(k, t)+\boldsymbol{\Omega}^{2}(k) \int d t^{\prime} \boldsymbol{M}\left(t-t^{\prime}\right) \dot{\boldsymbol{S}}\left(k, t^{\prime}\right)=0,
$$

where $\boldsymbol{\Omega}^{2}(k)=\frac{k^{2} k_{B} T}{m} \boldsymbol{S}^{-\mathbf{1}}(k)$ and $\boldsymbol{S}(k, t)$ is the matrix of intermediate scattering functions $S_{\alpha \beta}(k, t)$ and the memory function $\mathcal{M}$ can be written as :

$$
\begin{array}{r}
\left(\Omega^{2}(k) \mathcal{M}(k, t)\right)_{\alpha \beta}=\frac{1}{2 \rho \sqrt{x_{\alpha} x_{\beta}}} \sum_{l l^{\prime} m m^{\prime}} \int \frac{d \boldsymbol{q}}{(2 \pi)^{3}} V_{\alpha l m}(\mathbf{q}, \mathbf{k}-\mathbf{q}) V_{\beta l^{\prime} m^{\prime}}(\mathbf{q}, \mathbf{k}-\mathbf{q}) \\
\times S_{m m^{\prime}}(|\mathbf{k}-\mathbf{q}|) S_{l l^{\prime}}(q) \phi_{m m^{\prime}}(|\mathbf{k}-\mathbf{q}|, t) \phi_{l l^{\prime}}(q, t),
\end{array}
$$

where $\phi_{\alpha \beta}(k, t)=\frac{S_{\alpha \beta}(k, t)}{S_{\alpha \beta}(k, 0)}, \mathbf{k}-\mathbf{q}=\mathbf{p}$ and $V_{\alpha l m}(\mathbf{q}, \mathbf{p})=$ $\left[\hat{\mathbf{k}} \cdot \mathbf{q} \delta_{\alpha m} C_{\alpha l}(q)+\hat{\mathbf{k}} \cdot \mathbf{p} \delta_{\alpha l} C_{\alpha m}(p)\right]$, here $\boldsymbol{C}(q)$ is defined as $\boldsymbol{S}^{-\mathbf{1}}(q)=\mathbf{1}-\boldsymbol{C}(q)$. The static structure factor is obtained from computer simulation.

Solving Eq.13 we have calculated the relaxation times, $\tau_{\alpha \beta}$ from $\phi_{\alpha \beta}(q, t)$ at $1 / e$ and the average relaxation time is obtained from the following equation,

$$
\tau_{M C T}=\sum_{\alpha \beta} x_{\alpha} x_{\beta} \tau_{\alpha \beta}
$$

\section{RESULT AND DISCUSSION}

In this section using simulation results, we discuss the role of entropy in determining the dynamics via the Adam Gibbs relationship. We also investigate the role of pair correlation function in the dynamics by calculating the pair configurational entropy from radial distribution function. All these studies are done for LJ and WCA systems at different densities. 


\section{A. Adam Gibbs relation}

The relaxation time and diffusion coefficient obtained from simulation can be well fitted to VFT form using divergence temparature, $T_{V F T}$ and kinetic fragility, $K_{V F T}$ as fitting parameters. In Fig.1 we show that within the density range studied here, the LJ system is more fragile than the WCA system. The difference between kinetic fragility for LJ and WCA system is maximum at $\rho=1.2$ and as density increases the difference decreases. In Table I the data shows that similar to $K_{V F T}$, the divergence temperature $T_{V F T}$ increases with density and the difference between $T_{V F T}$ values for LJ and WCA systems reduces as density increases.
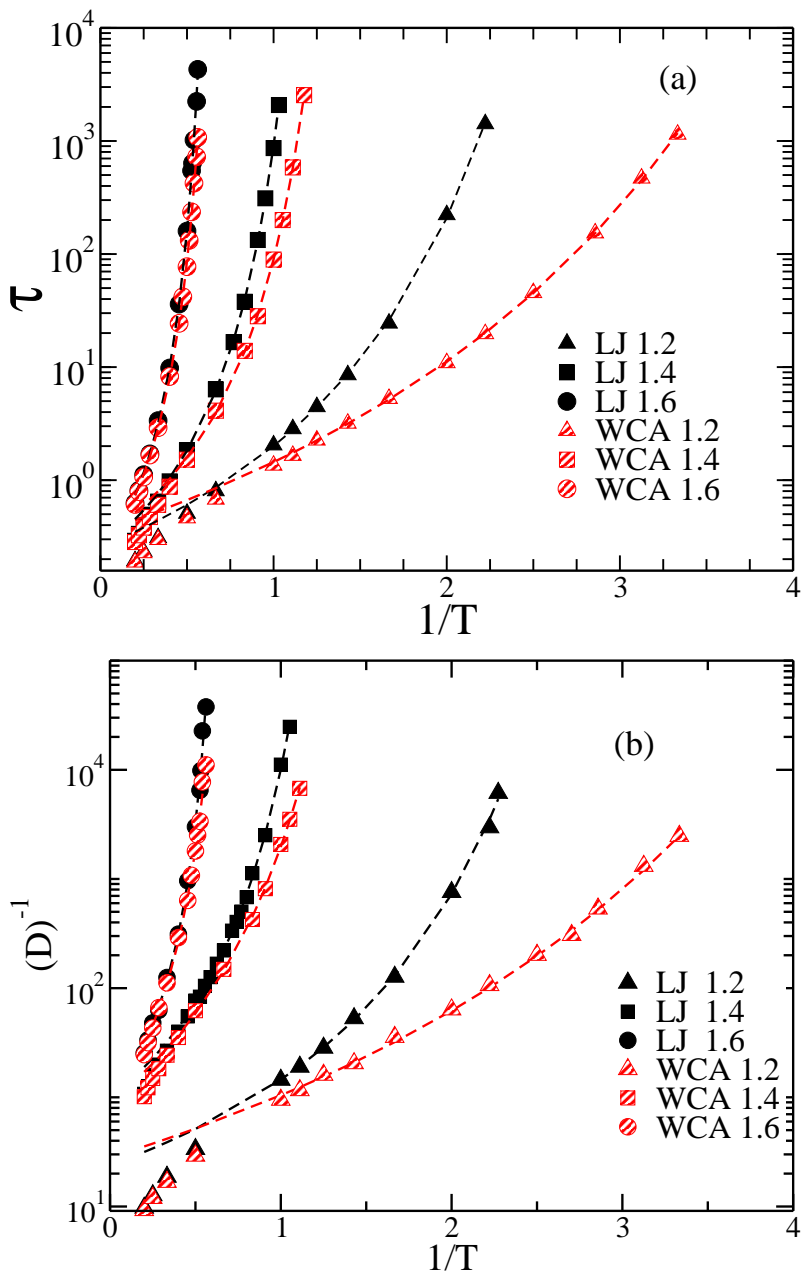

FIG. 1. (a)The VFT fit of the relaxation time $\tau$ defined from overlap function $\langle q(t=\tau)\rangle=1 / e$. The plot shows LJ system is more fragile than WCA system. At higher densities the difference in dynamics between LJ and WCA systems decreases. (b) The VFT fit of inverse of diffusion coefficient shows qualitatively similar behaviour as found for relaxation time. The values of kinetic fragilities from both the quantities have been given in the Table I.

In an earlier study we have shown that the kinetic fragility both for LJ and WCA systems at $\rho=1.2$ is
TABLE I. The values of kinetic fragility $\left(K_{V F T}\right)$ and the divergence temperature $\left(T_{V F T}\right)$ for all three densities are tabulated below. At higher density both the values of $K_{V F T}$ and $T_{V F T}$ come closer for LJ and WCA systems which indicate that the dynamical differences between the systems decrease at higher density .

\begin{tabular}{|c|c|c|c|c|}
\hline$\rho$ & $\begin{array}{c}K_{V F T}^{L J} \\
\text { from } \tau\end{array}$ & $\begin{array}{c}K_{V F T}^{W C A} \\
\text { from } \tau\end{array}$ & $\begin{array}{c}K_{V F T}^{L J} \\
\text { from D }\end{array}$ & $\begin{array}{c}K_{V F T}^{W C A} \\
\text { from D }\end{array}$ \\
\hline 1.2 & 0.187 & 0.124 & 0.151 & 0.116 \\
\hline 1.4 & 0.255 & 0.237 & 0.209 & 0.166 \\
\hline 1.6 & 0.291 & 0.285 & 0.284 & 0.257 \\
\hline \hline$\rho$ & $\begin{array}{c}T_{V F T}^{L J} \\
\text { from } \tau\end{array}$ & $\begin{array}{c}T_{V F T}^{W C A} \\
\text { from } \tau\end{array}$ & $\begin{array}{c}T_{V F T}^{L J} \\
\text { from D }\end{array}$ & $\begin{array}{c}T_{V F T}^{W C A} \\
\text { from D }\end{array}$ \\
\hline 1.2 & 0.278 & 0.150 & 0.239 & 0.132 \\
\hline 1.4 & 0.678 & 0.579 & 0.592 & 0.471 \\
\hline 1.6 & 1.319 & 1.258 & 1.255 & 1.153 \\
\hline
\end{tabular}

related to the thermodynamic fragility ${ }^{27}$. In a similar fashion, here we analyze the relationship between kinetic and thermodynamic fragility over a wider range of densities. We compute configurational entropy at different densities. Thermodynamic integration is used to calculate the total entropy and subtracting the vibrational entropy from it (details given in Sec. IIID) we obtain configurational entropy. For all the systems studied, $T S_{c}$ show a linear behaviour when plotted with temperature. We calculate the Kauzmann temperature, $T_{K}$, by extrapolation of this plot (Fig.2). The values are similar to the corresponding $T_{V F T}$ values (Table II). Further, the thermodynamic fragility $K_{T}$, which is the slope of $T S_{c}$ vs. $T / T_{K}$ plot, can be computed. We find that $K_{T}$ has a strong density dependence and increases with density. However similar to that found in the case of kinetic fragility, the difference between LJ and WCA systems in terms of thermodynamic fragility also decreases at higher densities (Table II).

TABLE II. The values of thermodynamic fragility and Kauzmann temperature for all three densities are tabulated below. At higher density both the values of $K_{T}$ and $T_{K}$ come closer for the LJ and the WCA systems. AG coefficients (both from relaxation time, $A^{\tau}$ and diffusion coefficient, $A^{D}$ ) for LJ and WCA systems have also been tabulated here. The AG coefficient increases with increase of density. The values of $K_{A G}=K_{T} / A^{\tau}$ are also tabulated here.

\begin{tabular}{|c|c|c|c||c|c|c|}
\hline$\rho$ & $K_{T}^{L J}$ & $K_{T}^{W C A}$ & $T_{K}^{L J}$ & $T_{K}^{W C A}$ \\
\hline 1.2 & 0.3084 & 0.1792 & 0.27 & 0.134 \\
\hline 1.4 & 0.723 & 0.609 & 0.631 & 0.50 \\
\hline 1.6 & 1.307 & 1.270 & 1.18 & 1.11 \\
\hline \multicolumn{1}{|c|}{} & $A_{L J}^{\tau}$ & $A_{W C A}^{\tau}$ & $A_{L J}^{D}$ & $A_{W C A}^{D}$ & $K_{A G}^{L J}$ & $K_{A G}^{W C A}$ \\
\hline 1.2 & 1.87 & 1.89 & 1.50 & 1.51 & 0.165 & 0.095 \\
\hline 1.4 & 3.57 & 4.37 & 2.63 & 3.30 & 0.202 & 0.139 \\
\hline 1.6 & 6.96 & 7.57 & 5.45 & 5.84 & 0.187 & 0.167 \\
\hline
\end{tabular}



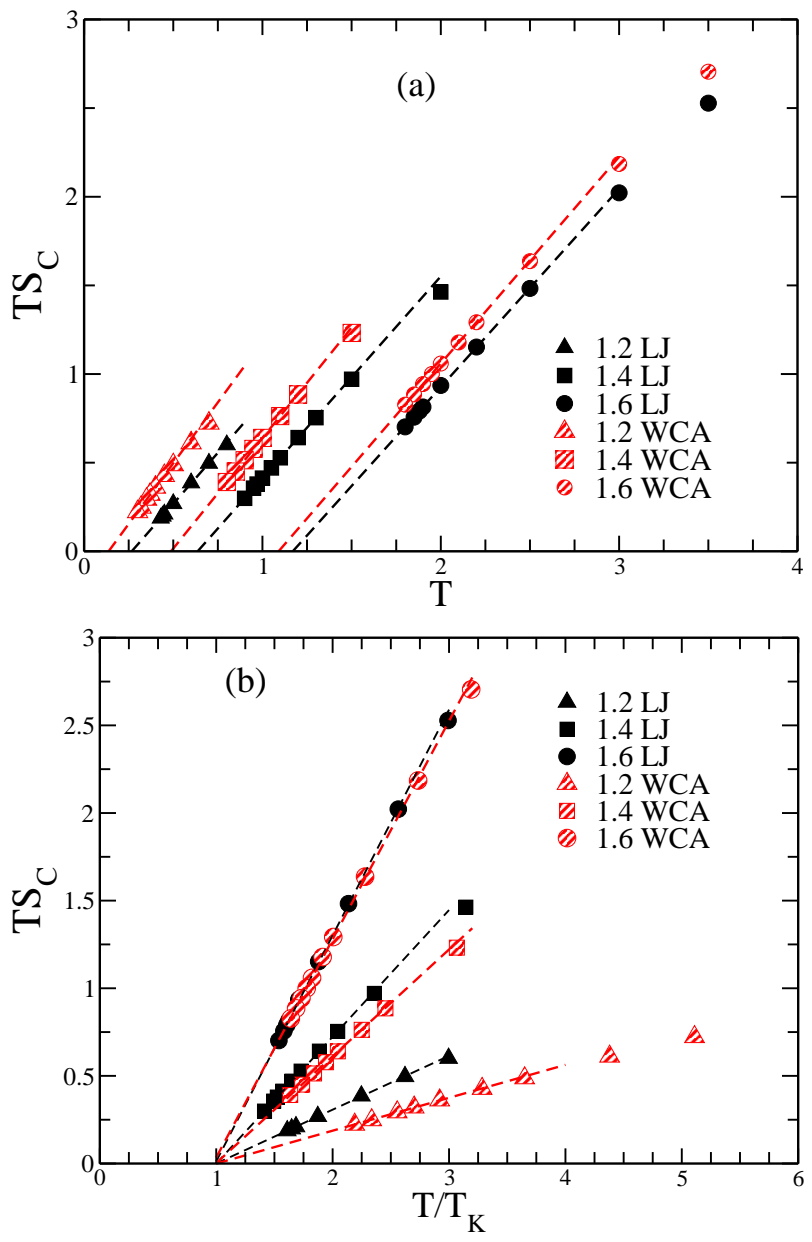

FIG. 2. (a) Temperature dependence of $T S_{c}$ for the studied models to determine the Kauzmann temperature. (b) $T S_{c}$ vs. $T / T_{K}$ for all the densities for both the systems. The thermodynamic fragility, $K_{T}$, is determined from the slope of the plot. $T_{K}$ and $K_{T}$ values are given in Table II for all the systems. Both the plot show that at high densities the difference between the LJ and WCA systems decrease.

Next we study the validity of the Adam Gibbs relation which connects the thermodynamics to the dynamics and find it to be valid for all the systems studied here. Fig. 3 shows the Adam-Gibbs plot both for $\tau$ and $D^{-1}$. Thus our study shows that the temperature variation of the configurational entropy $S_{c}$ captures the dynamics and also the differences in the dynamics between the LJ and the WCA systems. The Adam Gibbs coefficient (A) also has a strong density dependence and increases with density (Table II). An earlier study has shown that the Adam Gibbs coefficient (A) is related to the high temperature activation energy $(\mathrm{E})^{43}$. It has also been found that ' $\mathrm{E}$ ' increases with increase in density ${ }^{4,55}$. Thus our finding of increase in ' $A$ ' value with density supports the earlier studies.

Note that we also find that although the thermodynamic fragility and the Adam Gibbs coefficients have strong density dependence, their ratio (Table II) which is
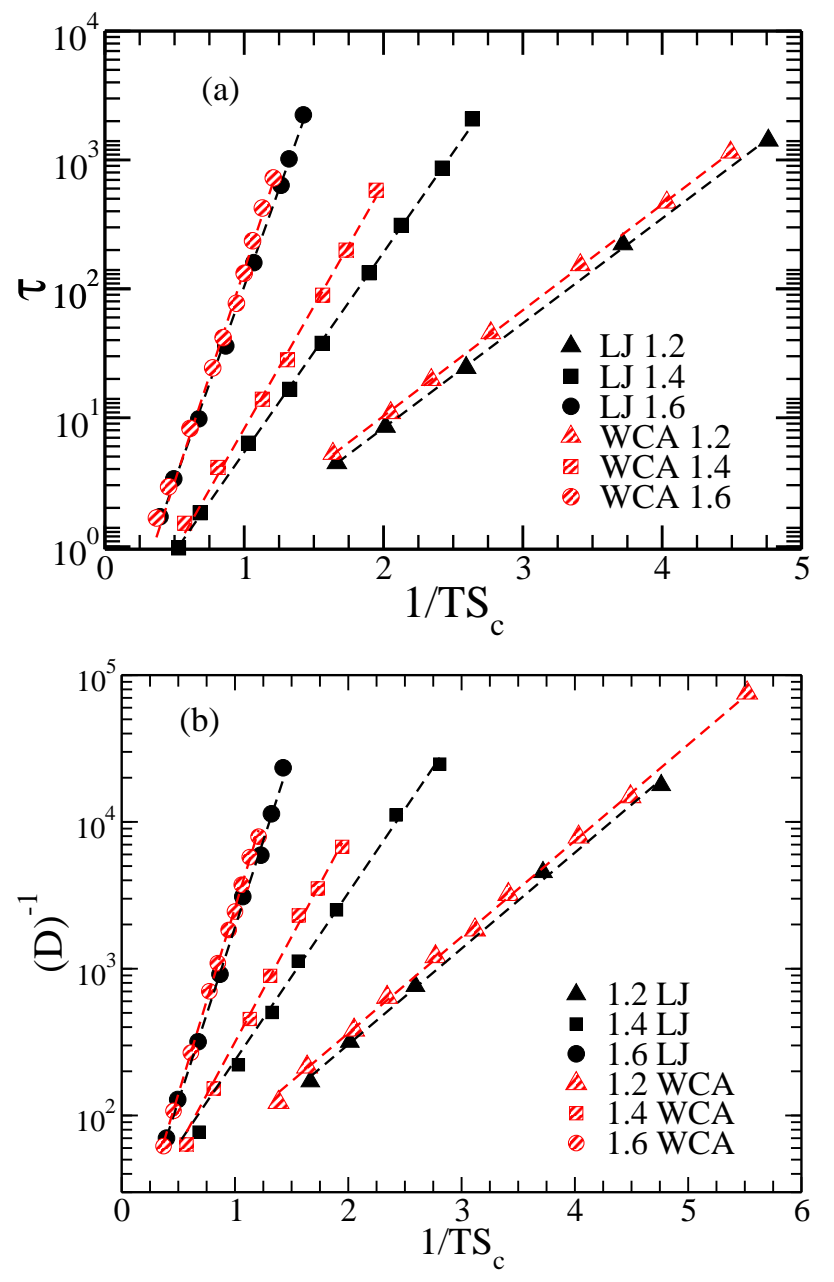

FIG. 3. The Adam-Gibbs plot in terms of (a) relaxation time and (b) diffusion coefficients. Both the plots show that the temperature dependence of dynamical quantities are quantitatively captured by the temperature variation of the configurational entropy.

related to kinetic fragility (see Sec. IIIC) shows a weak dependence on density. This kind of observation on density dependence has already been reported for the LJ system $^{6,56}$. However although unlike the LJ system, the WCA system does not show density-temperature scaling and thus density independence of fragility ${ }^{6}$, compared to the thermodynamic fragility the density dependence of the kinetic fragility even in WCA system is found to be weak (Fig.4).

Note that from Fig.2a we find that at same temperature, the configurational entropy is different for the two systems. Since $S_{c}$ has two different components, the total entropy and the vibrational entropy, it would be interesting to understand which part of the entropy gives rise to the difference in $S_{c}$. 


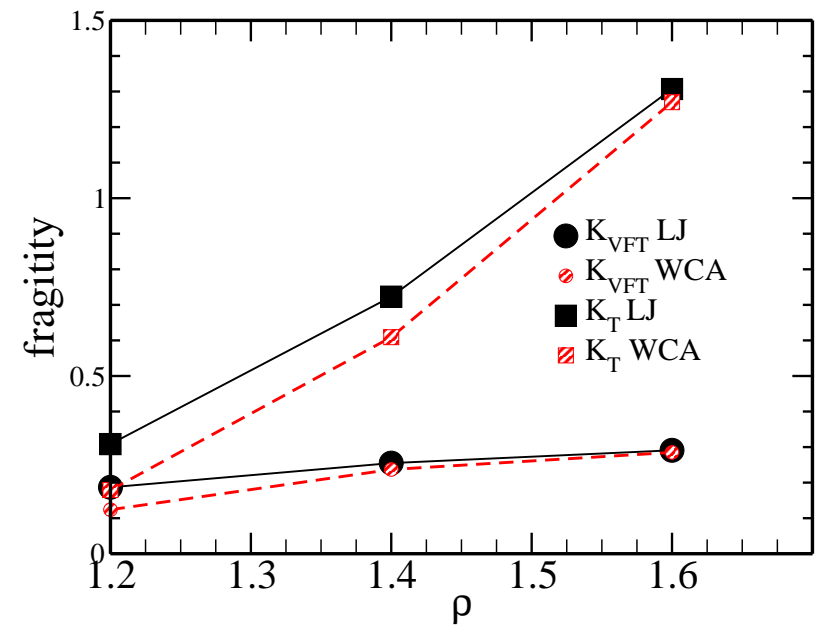

FIG. 4. Density dependence of thermodynamic and kinetic fragilities. Thermodynamic fragilities show strong temperature dependence, kinetic fragilities show weak temperature dependence for both LJ and WCA systems.

\section{B. Density of states and vibrational entropy}

In this section, we analyse the vibrational density of states of the LJ and the WCA systems. The vibrational entropy is calculated from vibrational density of states (VDOS), $P(\omega)=(1 / 3 N)<\sum_{l} \delta\left(\omega-\omega_{l}\right)>$, where $\omega_{l}$ is the frequency of the mode l. Normal modes of vibration are obtained by diagonalizing the Hessian matrix using $\mathrm{LAPACK}^{57}$. We find that similar to what has been reported earlier ${ }^{22}$, the difference between VDOS for the LJ and WCA systems are maximum at $\rho=1.2$ and as density increases they come closer, as a result of which at $\rho=1.6$, VDOS more or less overlap (Fig.5a). However in the calculation of $S_{v i b}$ we find that the difference between the two systems is negligible through out the density range studied here. At $\rho=1.2$ although VDOS are different, because of the cancellation of the values at low and high frequency of VDOS the $S_{v i b}$ appears to be similar. The WCA system has more low frequency modes and at $\rho=1.2$ we find some rattlers with zero frequency modes whose contribution has been ignored in the present study.

\section{Entropy : pair and higher order}

In the above section, we have shown that $S_{v i b}$ is similar for LJ and WCA systems in all three densities. This implies that the difference in $S_{c}$ comes from the total entropy. In that, the $S_{i d}$ is same for the LJ and WCA systems at a fixed density and temperature, thus it is the excess entropy which is responsible for the difference. Fig.6a shows indeed the $S_{e x}$ of the two systems are different and as density increases the difference in $S_{e x}$ between LJ and WCA systems decreases. This observation again supports our finding that at higher density both the sys-
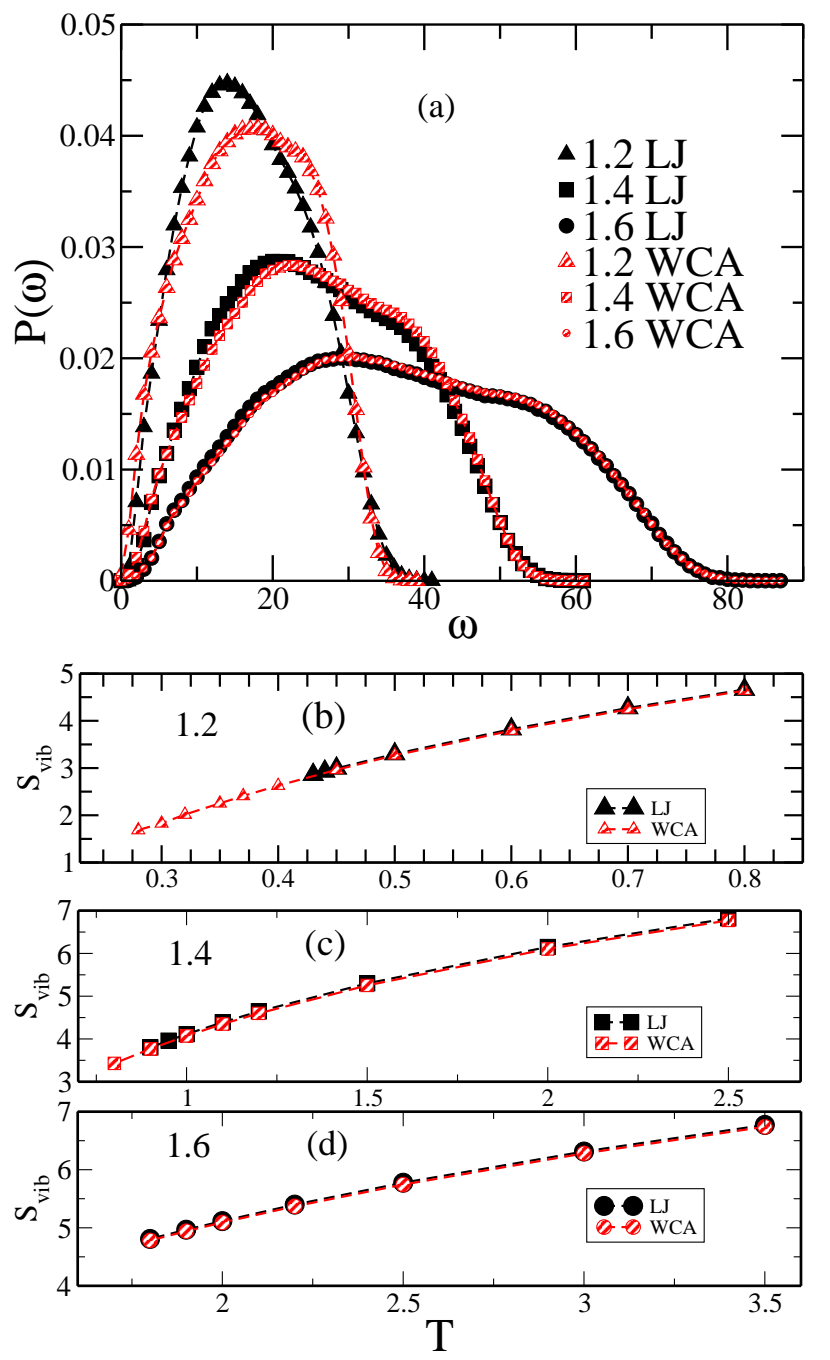

FIG. 5. (a) Distribution of density of vibrational states , $P(\omega)$, for $1.2,1.4,1.6$ density $(\mathrm{T}=0.45,1.00,1.90$ respectively). At $\rho=1.2$ the difference in the distribution of $P(\omega)$ for LJ and WCA system is maximum. At higher density the distributions are almost overlapping for LJ and WCA systems. The temperature dependence of vibrational entropy for (b) 1.2 , (c) 1.4 , (d)1.6 density. $S_{v i b}$ hardly shows any difference between LJ and WCA systems.

tems thermodynamically behaves in a similar way.

As given in Sec. IIIE, the $S_{e x}$ can be separated into pair and higher order terms. We find that both the $S_{2}$ and $\Delta S$ are different for LJ and WCA systems. As it has been already shown that the higher order terms are different for the two systems ${ }^{9}$, thus the difference in $\Delta S$ is not unexpected. However since the pair structures are similar, the difference in pair entropy is not intuitive. But our study shows that the difference in $S_{e x}$ is driven by $S_{2}$ as the contribution from $\Delta S$ is really small (note the y-axis scale of Fig.6b and Fig.6c). We find that similar to $S_{e x}$, the maximum difference in $S_{2}$ is present at 1.2 density. As density increases the difference between the two systems in $S_{2}$ and $\Delta S$ decreases. 

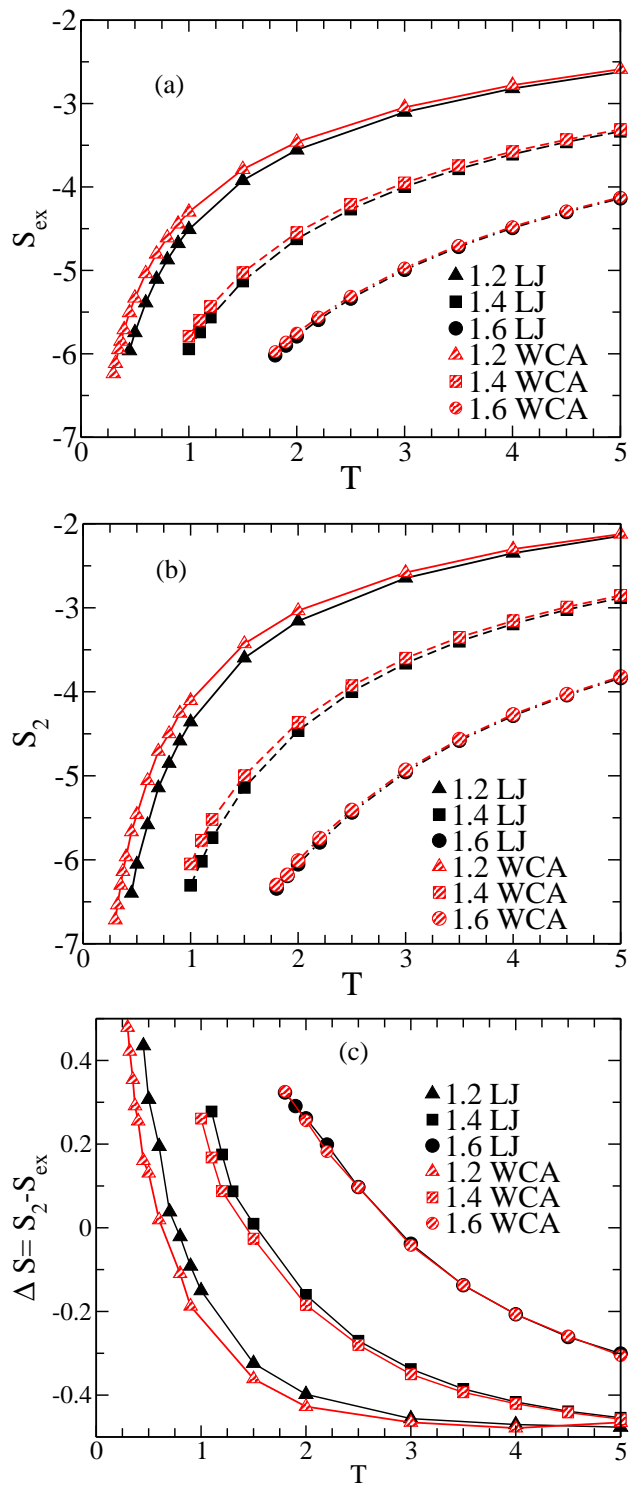

FIG. 6. (a) $S_{e x}$, calculated from thermodynamic integration, with temperature for LJ and WCA systems at different densities. (b) Pair entropy $\left(S_{2}\right)$ calculated from pair correction function using Eq.11 for all the systems. Both $S_{e x}$ and $S_{2}$ have negative values and decreases at lower temperature. (c) The temperature variation of residual multiparticle entropy $(\Delta S)$. All the plots show that at higher densities the difference between LJ and WCA systems decrease.

Next we analyse the origin of the difference in $S_{2}$ of the two systems. Although the total radial distribution function of LJ and WCA systems look similar ${ }^{3}$, in Fig.6b we find that pair entropy for LJ and WCA systems are quite different and the difference is maximum at $\rho=1.2$. However it has been earlier shown that the partial radial distribution functions are not identical at this density ${ }^{25}$ and the radial distribution function of the B-type particles are indeed quite different (Fig.7c inset). So the difference in pair entropy is expected to arise from the rdf of B-type particles. Also it is imperative to under-
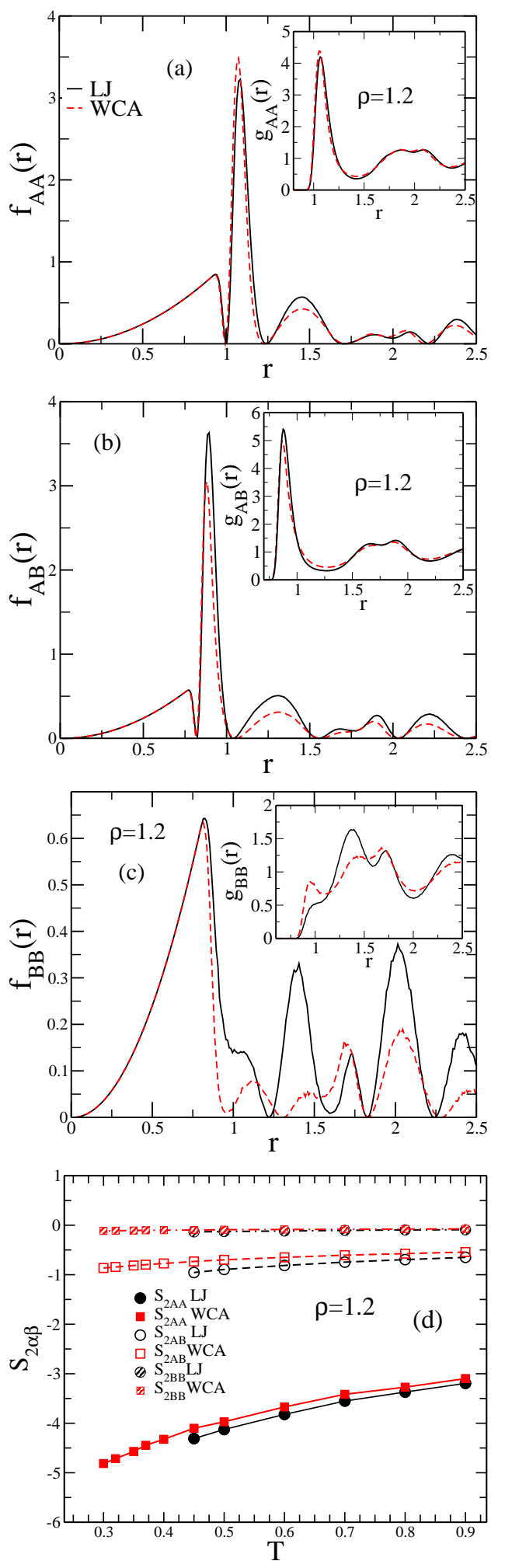

FIG. 7. (a) Comparison of the ' $\mathrm{r}$ ' dependence of $f_{A A}(r)$ (Eq.17) for LJ and WCA systems. (inset) Radial distribution function for A-type particles. (b) Similar plot for AB and (c) BB pair. All the plots show that the small difference in rdf peak and minima position amplify in the entropic terms $\left(f_{\alpha \beta}(r)\right)$. (d) The partial contribution of AA, AB, BB type particles to the total entropy. This plot shows that although rdf and $f_{B B}(r)$ for B-type particles have maximum difference between LJ and WCA systems, the difference in pair entropy comes from $\mathrm{AA}$ and $\mathrm{AB}$ pairs. All the plots are given for $\rho=1.2$. 
stand if the difference comes from the short or long range part of the rdf. As the difference in $S_{2}$ is maximum at $\rho=1.2$, we choose this density for our next analysis and address these two points mentioned above.

The pair entropy $S_{2}$ for a binary system can be written in terms of the partial radial distribution functions,

$$
\frac{S_{2}}{k_{B}}=\sum_{\alpha, \beta} S_{2 \alpha \beta}=-2 \pi \rho \sum_{\alpha, \beta} x_{\alpha} x_{\beta} \int_{0}^{\infty} f_{\alpha \beta}(r) d r,
$$

where,

$$
f_{\alpha \beta}(r)=\left\{g_{\alpha \beta}(r) \ln g_{\alpha \beta}(r)-\left[g_{\alpha \beta}(r)-1\right]\right\} r^{2} .
$$

Here $g_{\alpha \beta}(r)$ is the atom-atom pair correlation between atoms of type $\alpha$ and $\beta, \rho$ is the density of the system, $x_{\alpha}$ is the mole fraction of component $\alpha$ in the mixture, and $k_{B}$ is the Boltzmann constant.

$S_{2 \alpha \beta}$ is the partial pair entropy for a binary system given by,

$$
\frac{S_{2 \alpha \beta}}{k_{B}}=-2 \pi \rho x_{\alpha} x_{\beta} \int_{0}^{\infty} f_{\alpha \beta}(r) d r .
$$

The plot of $f_{\alpha \beta}(r)$ vs. $r$ shows that the difference mainly comes from the first few peak and minima positions of the rdf and not from large r (Fig.7a, Fig.7b, Fig.7c). We also find that although $g_{A A}(r)$ and $g_{A B}(r)$ (inset of Fig.7a, Fig.7b) for both the systems are very similar, the entropic terms $\left(f_{\alpha \beta}(\mathrm{r})\right)$ for $\mathrm{AA}$ and $\mathrm{AB}$ pairs show enough difference. More interestingly, we find that although as a reflection of $g_{B B}(r)$, the $f_{\alpha \beta}(r)$ of B-B type of particles looks quite apart, but it has negligible effect on $S_{2 B B}$. The small mole fraction of B-type of particles, minimizes the differences (Fig.7d). The primary contribution to the difference in $S_{2}$ between the LJ and WCA systems come from $S_{2 A A}$ and $S_{2 A B}$. Thus, our study shows that small difference at the two body level is properly amplified in $S_{2}$, which allows us to disentangle the LJ and WCA systems.

Next to calculate the effect of pair correlation on the entropy, we consider the separation of the configurational entropy into pair and many body parts as described earlier (Sec. IIIE) ${ }^{27}$. Using $S_{2}$ information we calculate the pair configurational entropy $S_{c 2}$, which goes to zero at the Kauzmann temperature $\left(T_{K 2}\right)$ given in the Table III. It has been earlier reported that this temperature has a connection to the MCT transition temperature ${ }^{55}$. The pair thermodynamic fragility $K_{T 2}$ is computed from the slope of $T_{S c 2}$ vs. $T / T_{K 2}$ plot. The plots show that the thermodynamic fragility even at the two body level is higher for the LJ system. Like relaxation time and thermodynamic fragility we find that the difference in pair thermodynamic fragility between LJ and WCA systems also decreases as density increases.

\section{Dynamics predicted by pair correlation}

Once we have determined the role of pair correlation in entropy, we now determine its effect on the low tem-

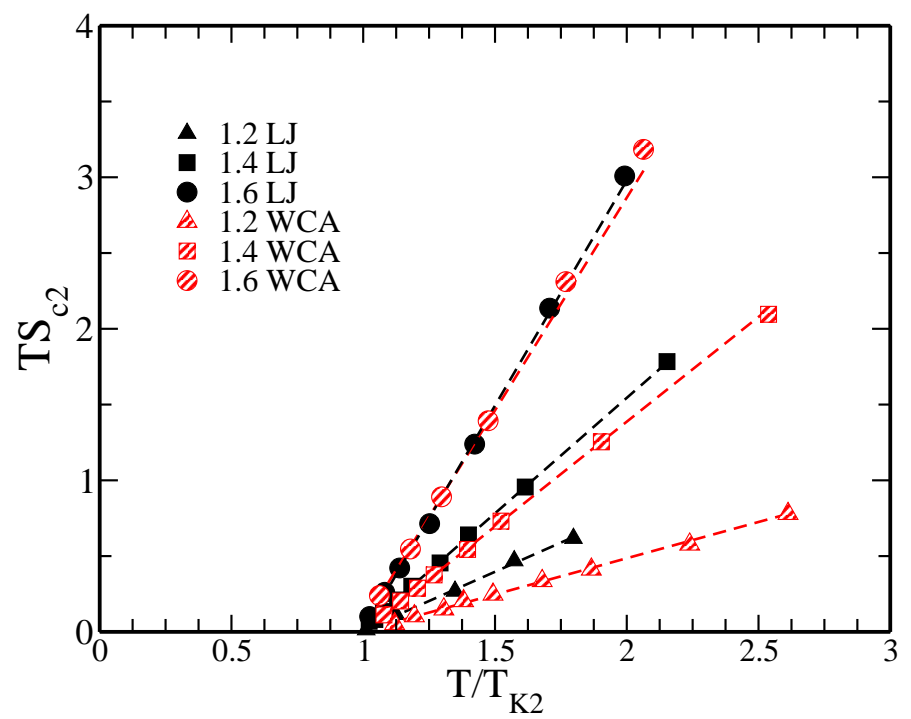

FIG. 8. $T S_{c 2}$ vs. $T / T_{K 2}$ plot. Determination of pair thermodynamic fragility, $K_{T 2}$ from the slope of the linear fit for all the systems. The temperature $\left(T_{K 2}\right)$ where $S_{C 2}$ goes to zero, is tabulated in Table III.

TABLE III. The values of pair thermodynamic fragility and pair Kauzmann temperature for all three densities are tabulated below. At higher density both the values of $K_{T 2}$ and $T_{K 2}$ come closer for LJ and WCA systems.

\begin{tabular}{|c|c|c||c|c|c|}
\hline$\rho$ & $K_{T 2}(L J)$ & $K_{T 2}(W C A)$ \\
\hline 1.2 & 0.795 & 0.483 & $\rho$ & $T_{K 2}(L J)$ & $T_{K 2}(W C A)$ \\
\hline 1.4 & 1.555 & 1.358 & 0.445 & 0.268 \\
\hline 1.6 & 2.971 & 2.936 & 1.4 & 0.929 & 0.788 \\
\hline 1.6 & 1.76 & 1.69 \\
\hline
\end{tabular}

perature dynamics. The AG relation can be re expressed $\mathrm{as}^{27}$,

$$
\begin{aligned}
\tau(T) & =\tau_{o} \exp \left(\frac{A}{T S_{c}}\right)=\tau_{o} \exp \left(\frac{A}{T S_{C 2}}-\frac{A \Delta S}{T S_{C 2} S_{C}}\right) \\
& =\tau_{2}^{A G} \exp \left(-\frac{A \Delta S}{T S_{C 2} S_{C}}\right)
\end{aligned}
$$

where $\tau_{2}^{A G}(T)=\tau_{o} \exp \left(\frac{A}{T S_{C 2}}\right)$. Note that the equalities in Eq.19 are exact as long as $T, S_{C 2}, S_{C}$ are finite. The $\tau_{2}^{A G}$ for the LJ and the WCA systems are plotted in Fig.9 for all the densities. The density and system dependence of $\tau_{2}^{A G}$ also shows similar characteristics as the simulated relaxation time, $\tau$. At lower density $\tau_{2}^{A G}$ of the two systems are far apart and as $\rho$ increases, they come closer. We also find that for a fixed density, $\tau_{2}^{A G}$ diverges at higher temperature compared to the actual relaxation time. Although this behaviour is reminiscent of MCT prediction $^{4}$, in the following part of the discussion we will show that this theory works in a regime where the MCT and other microscopic theories which rely on the paircorrelation $(g(r))$ fail to capture the difference between the dynamics of the two systems ${ }^{4,7,8}$. The pair kinetic fragility , $K_{V F T 2}$, as obtained by fitting the temperature 
dependence of $\tau_{2}^{A G}$ to a VFT form, shows that the LJ system is more fragile. If we compare the pair fragility to the total fragility of the system we find that both for the kinetic and thermodynamic fragilities the pair one predicts a higher value. Usually the fragility of a system is connected to many body interaction ${ }^{58}$. However our analysis shows a connection of fragility to the two body correlations which is similar to a recent observation by Wang et $a t^{23}$.

In an earlier work by some of us performed at a fixed density, we have shown that in contradiction to conventional wisdom the divergence in dynamics and also the difference between the dynamics in the LJ and WCA systems are driven by pair correlation. Our present study over a wider range of density confirms this as a generic behaviour independent of density of the systems.

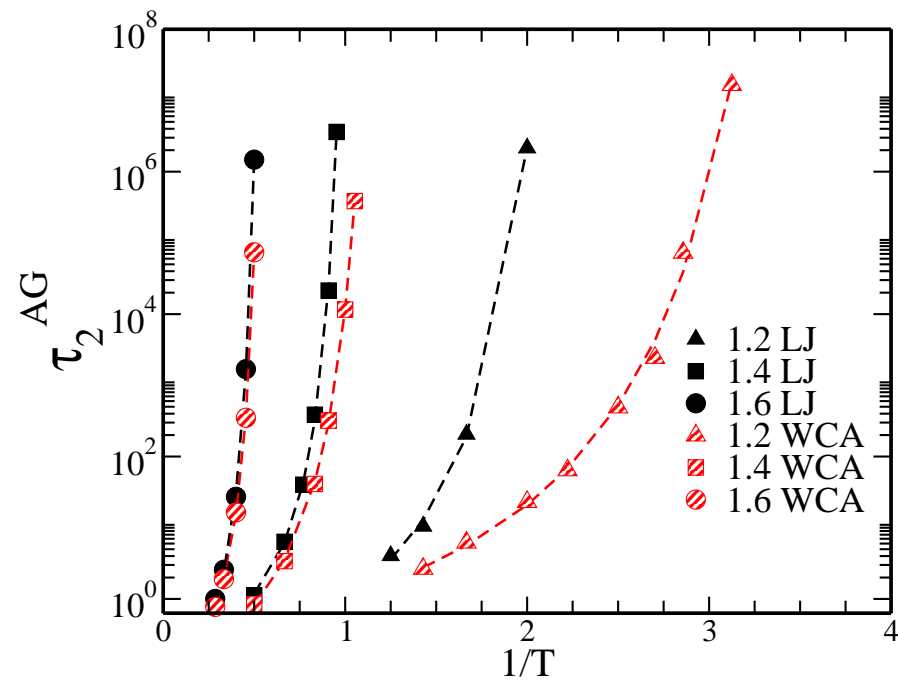

FIG. 9. The temperature dependence of $\tau_{2}^{A G}$ for all the systems. The difference in the dynamics for LJ and WCA systems is driven by two-body correlation. Similar to the actual relaxation time, the plot shows the difference decreases as density increases.

We next focus on $\rho=1.6$ where the $\tau_{M C T}$ (calculation details given in Sec. IIIF) for both the systems almost overlap. In Fig.10 we show that even at this density $\tau_{2}^{A G}$ values are different and the difference is more than that found for the total relaxation time. A probable reason for the AG theory, only with pair correlation, $S_{2}$, to work better than the MCT is that the AG relation uses the entropy. In the calculation of the two body entropy $S_{2}$ although we consider only the radial distribution function but in effect it calculates a many body quantity. This in turn magnifies the effect observed at the two body level.

However although $\tau_{2}^{A G}$ as compared to $\tau_{M C T}$ is shown to be better in predicting the difference between the two systems, it shows a divergence at a higher temperature reminiscent of the behaviour of $\tau_{M C T}$. Surprisingly the two behaviours are similar in trend but they are fundamentally different, where the AG comes from an activation picture whereas MCT in the form presented here is a mean field theory at the two body level without any activation. In a recent work by us it was shown that those two fundamentally different theories predict the correct temperature dependence of relaxation time in a certain temperature region ${ }^{55}$. However it has also been shown that the AG theory at the two body level cannot predict the MCT power law behaviour. Even above $T_{c}$, the residual multiparticle entropy plays an important role and below $T_{c}$, RMPE determines the dymanics fully. These findings provide the evidence that multiparticle correlations are essential to correctly describe the physics of supercooled liquids. The effect of density on multiparticle correlation will be addressed in future work.

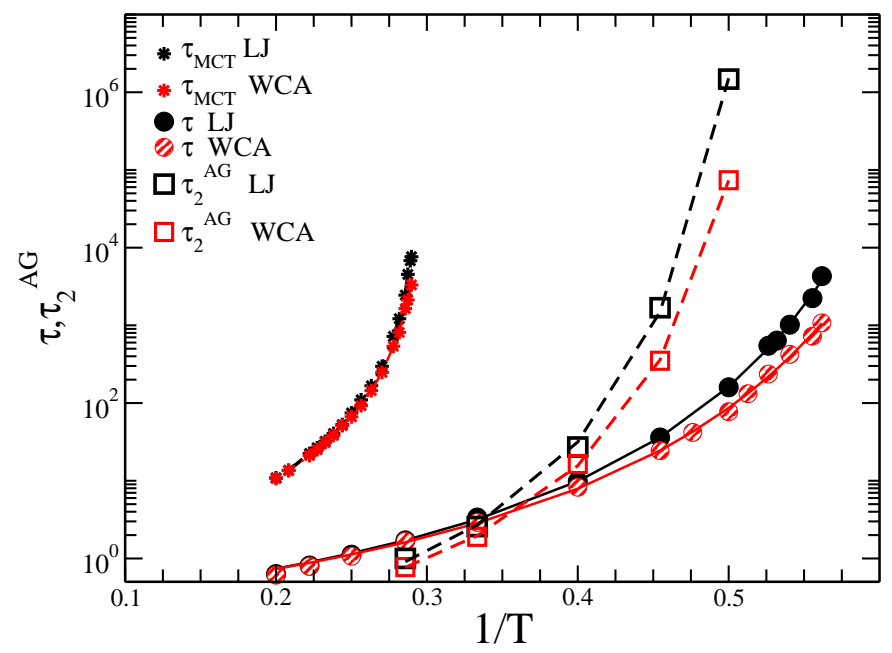

FIG. 10. $\tau, \tau_{2}^{A G}, \tau_{M C T}$ vs. $1 / T$ for LJ and WCA systems at $\rho=1.6$. Unlike MCT relaxation time, the estimated $\tau_{2}^{A G}$ shows a difference in the dynamics of LJ and WCA systems.
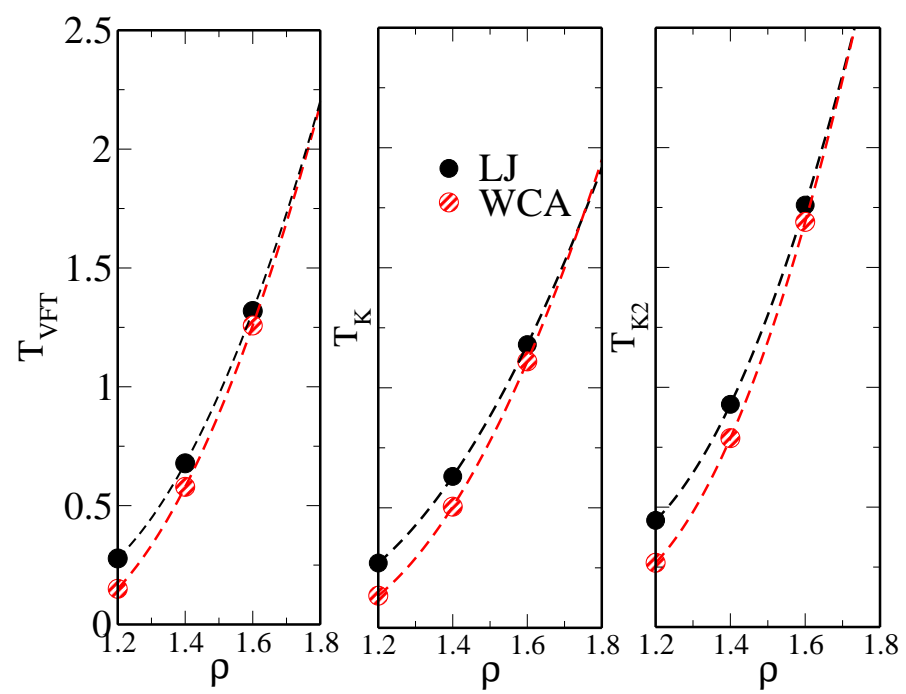

FIG. 11. Density dependence of $T_{V F T}, T_{K}, T_{K 2}$ have been fitted and extrapolated to a higher density, which show all three temperatures predicts that LJ and WCA systems will behave in a similar way around $\rho=1.8$. 


\section{E. Phase diagram from thermodynamics and kinetics}

In this section by analyzing the density dependence of the different divergence temperatures calculated in the previous sections, we make an attempt to predict the density where the thermodynamical and dynamical properties of both the LJ and WCA systems will be similar. The density dependence of the divergent temperatures behave in a similar fashion. As mentioned earlier $T_{V F T}, T_{K}, T_{K 2}$ values come closer as density increases (Fig.11). If we fit the data and extrapolate it to higher densities, we find that all the three temperatures predict that the WCA and LJ system will behave in a similar way around $\rho \simeq 1.8$. Wang et. al have shown that above $\rho=1.9 \pm 0.1$ the structural and vibrational quantities of the glasses show scaling collapse ${ }^{22}$. Another study by Wang et. al have shown that at high density $(\rho \simeq 1.8)$ both the LJ and WCA systems have similar structure, fragility and dynamics ${ }^{23}$. The MCT critical temperature, which has been earlier connected to the pair Kauzman temperature $^{55}$, also shows that at higher densities the difference decreases ${ }^{3}$. Dyre and coworkers have claimed that the inclusion of interactions of all first coordination shell neighbours ${ }^{24}$, can make LJ and WCA systems similar. This however predicts a density which is higher than that predicted by the VDOS or the divergent temperatures. Note that the density predicted by all the different divergence temperatures are similar. Our finding, that above a certain density the dynamics and thermodynamics of both LJ and WCA systems will behave in a similar manner, is consistent with the observation made earlier ${ }^{3,22-24}$.

\section{CONCLUSION}

In this work, we study a wide range of densities and show that the difference in dynamics for the LJ and thr WCA systems is a manifestation of their thermodynamical difference. As density increases the differences in the dynamical and thermodynamical quantities of the LJ and the WCA systems decrease, as a result of which the fragilities and the transition temperatures come closer at higher densities. The well known Adam Gibbs relation, which connects the thermodynamic and kinetic fragilities, is found to be valid for all the densities and systems studied here. Our study shows that although the total pair correlation function for the LJ and the WCA systems are similar, the pair configurational entropy and corresponding dynamics show a large difference. Our analysis from partial radial distribution function and partial pair entropy show that despite the maximum difference in $\mathrm{BB}$ rdf, due to the lower mole fraction of B type of particles, the difference in pair entropy comes from $\mathrm{AB}$ and $\mathrm{AA}$ pairs. We also find that the difference in the pair entropy arises from the short range part of the correlation functions. The density dependence of the pair configurational entropy and the two body relaxation time follows the same trend as observed for the corresponding total quantities.

In an earlier work done at one particular density we have shown that the difference in thermodynamics between the LJ and the WCA systems and also the divergence of their dynamics are driven by pair correlation ${ }^{27}$. In this study, we show that this indeed is a generic behaviour independent of the density of the system. We find that both the thermodynamic and kinetic fragility predicted by pair correlation are larger than that predicted by the total correlation. Usually the fragility of a system is thought to be connected to cooperativity and thus to a collective behaviour ${ }^{9}$. However our analysis shows that the fragility is also connected to the two body correlation which is similar to a recent observation by Wang et $a l^{23}$.

The dynamics predicted by the AG relation at the two body level is reminiscent of the MCT behaviour predicting a divergence of the dynamics at a higher temperature. However our analysis at $\rho=1.6$ shows that unlike MCT the pair correlation entropy and the corresponding dynamics can predict the difference between the two systems. Thus AG relation at the two body level, although has the shortcomings of the MCT, fairs better than MCT in predicting the difference between the two systems. A probable reason for this may be the fact that entropy even when calculated at the two body level provides a many body quantity.

The analysis of the density dependence of the different divergent temperatures predict that around $\rho \simeq 1.8$ both systems will behave in a similar manner. This supports the earlier observations ${ }^{3,22-24}$. Our study although emphasizes the role of pair correlation, we also find that to describe the total dynamics pair correction is not enough and higher order terms are important.

\section{ACKNOWLEDGEMENTS}

This work has been supported by the Department of Science and Technology (DST), India and CSIR-MultiScale Simulation and Modeling project. AB thanks CSIR and MKN thanks UGC for fellowship. SS thanks the Sheikh Saqr Laboratory, JNCASR, for support.

${ }^{1}$ J. P. Hansen and I. R. McDonald, Elsevier, Amsterdam (1986).

2 J. D. Weeks, D. Chandler, and H. C. Andersen, J. Chem. Phys. 54, 5237 (1971).

${ }^{3}$ L. Berthier and G. Tarjus, Phys. Rev. Lett. 103, 170601 (2009).

${ }^{4}$ L. Berthier and G. Tarjus, Phys. Rev. E 82, 031502 (2010).

${ }^{5}$ L. Berthier and G. Tarjus, EPJE 34, 1 (2011).

${ }^{6}$ L. Berthier and G. Taruus, J. Chem. Phys. 134, (2011).

${ }^{7}$ K. S. Schweizer, J Chem. Phys. 123, (2005).

${ }^{8}$ K. S. Schweizer, J Chem. Phys. 123, (2005).

${ }^{9}$ D. Coslovich, Phys. Rev. E 83, 051505 (2011).

${ }^{10}$ D. Coslovich, J. Chem. Phys. 138, 12A539 (2013).

${ }^{11}$ D. Kivelson, S. A. Kivelson, X. Zhao, Z. Nussinov, and G. Taruus, Physica A 219, 27 (1995). 
${ }^{12}$ S. Karmakar, C. DASGupta, and S. SAStry, PNAS 106, 3675 (2009).

${ }^{13}$ S. Karmakar and I. Procaccia, Phys. Rev. E 86, 061502 (2012).

${ }^{14}$ S. K. Nandi and S. Ramaswamy, Phys. Rev. Lett. 109, 115702 (2012).

${ }^{15}$ A. Malins, J. Eggers, C. P. Royall, S. R. Williams, and H. TANaka, J. Chem. Phys. 138, 12A535 (2013).

${ }^{16}$ G. M. Hocky, T. E. Markland, and D. R. Reichman, Phys. Rev. Lett. 108, 225506 (2012).

${ }^{17}$ K. Kim and S. Saito, J. Chem. Phys. 138, 12 A506 (2013).

${ }^{18}$ C. Cammarota, A. Cavagna, G. Gradenigo, T. S. Grigera, and P. Verrocchio, J. Chem. Phys. 131, 194901 (2009).

${ }^{19}$ C. Cammarota, A. Cavagna, G. Gradenigo, T. S. Grigera, and P. Verrocchio, J. Chem. Phys. 131, 194901 (2009).

${ }^{20}$ C. Cammarota and G. Biroli, PNAS 109, 8850 (2012).

${ }^{21}$ R. L. Jack and J. P. Garrahan, J. Chem. Phys. 123, 164508 (2005).

${ }^{22}$ L. WANg and N. Xu, Phys. Rev. Lett. 112, 055701 (2014).

${ }^{23}$ L. WANG, P. GUAN, and W. H. WANG, arXiv:1602.08893v1.

${ }^{24}$ S. Tøxvaerd and J. C. Dyre, J. Chem. Phys. 135, 134501 (2011).

${ }^{25}$ U. R. Pedersen, T. B. Schrøder, and J. C. Dyre, Phys. Rev. Lett. 105, 157801 (2010).

${ }^{26}$ L. Bøhling, A. A. Veldhorst, T. S. Ingebrigtsen, N. P. BAiley, J. S. Hansen, S. Tøxvaerd, T. B. Schrøder, and J. C. Dyre, J Phys: Condens. Matter 25, 03210 (2013).

${ }^{27}$ A. Banerjee, S. Sengupta, S. Sastry, and S. M. Bhattacharyya, Phys. Rev. Lett. 113, 225701 (2014).

${ }^{28}$ G. Adam and J. H. GibBs, J. Chem. Phys. 43, 139 (1965).

${ }^{29}$ F. Sciortino, W. Kob, and P. Tartaglia, Phys. Rev. Lett. 83, 3214 (1999)

${ }^{30}$ S. SASTRY, Nature 409, 164 (2001).

${ }^{31}$ R. J. Speedy, J. Chem. Phys. 110, 4559 (1999).

${ }^{32}$ R. J. Speedy, J. Chem. Phys. 114, 9069 (2001).

${ }^{33}$ A. Angelani and G. Foffi, J. Phys.: Condens. Matter 19, 256207 (2007).

${ }^{34}$ A. Scala, F. W. Starr, E. L. Nave, F. Sciortino, and H. E. StANlEy, Nature(London) 406, 166 (2000).
${ }^{35}$ F. W. Starr, S. Sastry, E. La Nave, A. Scala, H. Eugene Stanley, and F. Sciortino, Phys. Rev. E 63, 041201 (2001).

${ }^{36}$ I. Saika-Voivod, F. Sciortino, and P. H. Poole, Phys. Rev. E 69, 041503 (2004).

${ }^{37} \mathrm{~S}$. Mossa, E. La Nave, H. E. Stanley, C. Donati, F. Sciortino, and P. Tartaglia, Phys. Rev. E 65, 041205 (2002).

${ }^{38}$ F. W. Starr, J. F. Douglas, and S. Sastry, J. Chem. Phys. 138 (2013).

${ }^{39}$ C. A. Angell, Nature 393, 521 (1998).

${ }^{40}$ K. Ito, C. T. Moynihan, and C. A. Angell, Nature 398 (1999).

${ }^{41}$ W. Kob and H. C. Andersen, Phys. Rev. E 51, 4626 (1995).

${ }^{42}$ S. J. Plimpton, J. Comput. Phys. 117, 1 (1995).

${ }^{43}$ S. Sengupta, F. Vasconcelos, F. Affoudrd, and S. Sastry, J. Chem. Phys. 135, 194503 (2011).

${ }^{44}$ S. Sastry, Phys. Rev. Lett. 85, 590 (2000).

${ }^{45}$ J. G. Kirkwood and E. M. Boggs, J. Chem. Phys. 10, 394 (1942).

${ }^{46}$ R. E. Nettleton and M. S. Green, J. Chem. Phys. 29, 1365 (1958).

${ }^{47}$ H. J. Raveché, J. Chem. Phys. 55, 2242 (1971).

${ }^{48}$ D. C. Wallace, J. Chem. Phys. 87, 2282 (1987).

${ }^{49}$ P. V. Giaquinta and G. Guinta, Physica A 187, 145 (1992).

${ }^{50}$ S. Prestipino and P. V. Giaquinta, J. Stat. Phys. 96, 135 (1999).

${ }^{51}$ F. Saija, S. Prestipino, and P. V. Giaquinta, J. Chem. Phys. 113, 2806 (2000).

52 F. Saija, S. Prestipino, and P. V. Giaquinta, J. Chem. Phys. 124, 244504 (2006)

${ }^{53}$ W. Götze, J. Phys.: Cond. Mat. 11, A1 (1999).

${ }^{54}$ E. Flenner and G. Szamel, Phys. Rev. E 72, 031508 (2005).

${ }^{55}$ M. K. Nandi, A. Banerjee, S. Sengupta, S. Sastry, and S. M. Bhattacharyya, J Chem. Phys. 143, 174504 (2015).

${ }^{56}$ S. Sengupta, T. B. Schrøder, and S. Sastry, Eur. Phys. J. E 36, 141 (2013).

${ }^{57}$ HTTP://WWW.NETLIB.ORG/LAPACK/.

${ }^{58}$ G. Taruus and C. Alba-Simionesco, arXiv:1401.2812 (2014). 